\title{
The UBR2 E3 ubiquitin ligase is over-expressed in brain metastatic breast cancer.
}

Shahan Mamoor ${ }^{1}$

1shahanmamoor@gmail.com

East Islip, NY USA

Post-translational modifications of proteins include the covalent conjugation of ubiquitin moieties to proteins by the catalytic function of $\mathrm{E} 3$ ligases ${ }^{1}$. We found that the brain metastases of patients with brain metastatic breast cancer express significantly higher levels of the UBR2 E3 ligase as compared to primary tumors of the breast, through mining of published microarray data ${ }^{2,3}$. The substrates of UBR2 ubiquitination may be important for the underlying biology of brain metastasis, and UBR2 could potentially represent a novel therapeutic target in a disease with limited treatment options.

Keyword: breast cancer, brain metastasis, central nervous system metastasis, ubiquitin, E3 ligase, UBR2, $\mathrm{N}$-end rule pathway, systems biology of breast cancer, targeted therapeutics in breast cancer. 
Patients treated with trastuzumab (Herceptin) face an incidence of central nervous system metastasis of $34 \% 4$. This alarmingly high frequency of CNS metastases events demands an enhanced understanding of the transcriptional makeup of brain metastatic tissues to support identification of therapeutic targets. We performed a global comparative analysis of transcription in the brain metastases of patients with brain metastatic breast cancer using published microarray data $a^{2,3}$. We discovered transcriptome-wide differential expression and over-expression of the UBR2 ubiquitin ligase in the brain metastatic tissues of patients with breast cancer.

\section{Methods}

We utilized microarray datasets GSE438372 and GSE100534³ for this differential gene expression analysis of brain metastatic breast cancer in conjunction with GEO2R. GSE43837 was generated using Affymetrix Human X3P Array technology with $n=19$ primary breast tumors and $n=19$ brain metastases from patients with breast cancer, analysis performed using platform GPL1352. GSE100534 was generated using Affymetrix Human Gene 1.0 ST Array technology with $n=16$ primary tumors from patients with breast cancer and $n=3$ brain metastases from patients with brain metastatic breast cancer. The Benjamini and Hochberg method of $p$-value adjustment was used for ranking of differential expression but raw $p$-values were used to assess statistical significance of global differential expression. Log-transformation of data was auto-detected, and the NCBI generated category of platform annotation was used. A statistical test was performed to evaluate whether UBR2 gene expression was significantly between primary breast tumors and brain metastases in humans with breast cancer using a two-tailed, unpaired t-test with Welch's correction. We used PRISM for all statistical analyses of differential gene expression in human breast cancer (Version 8.4.0)(455).

\section{Results}

We mined published microarray data 2,3 to identify genes most transcriptionally perturbed in the brain metastases of patients with brain metastatic breast cancer.

\section{UBR2 is differentially expressed in the brain metastases of patients with brain metastatic breast cancer.}

We identified UBR2 as among the genes whose expression was most significantly different in the brain metastases of patients with brain metastatic breast cancer when compared to primary tumors of the breast. When sorting each of the genes expressed in brain metastases based on significance of change as compared to primary tumors of the breast, UBR2 ranked 20 out of 61359 total transcripts (Table 1). Differential expression of UBR2 in brain metastatic breast cancer was statistically significant (Table 1; $p=0.0000678$ ).

We queried a second microarray dataset to validate transcriptome-wide differential of UBR2 in brain metastatic breast cancer. When sorting each of the genes expressed in brain metastases based on significance of change as compared to primary tumors of the breast, UBR2 ranked 7256 out of 33297 total transcripts (Table 2). Differential expression of UBR2 in brain metastatic breast cancer in this second dataset was statistically significant (Table $2 ; p=4.05 \mathrm{E}-02$ ).

UBR2 is expressed at significantly higher levels in the brain metastases of patients with brain metastatic breast cancer. 
We obtained exact mRNA expression levels of UBR2 to evaluate magnitude and direction of change in UBR2 gene expression between brain metastases and primary tumors of the breast. UBR2 was expressed at higher levels in brain metastases as compared to primary tumors of the breast, and this difference was statistically significant (Figure $1 ; p=0.0024$ ). We calculated a mean fold change of $2.82 \pm$ 2.14 in UBR2 expression in brain metastases relative to primary breast tumors in patients with breast cancer (Table 1).

Thus, we identified the E3 ubiquitin ligase UBR2 as among the genes whose expression was most significantly different transcriptome-wide when comparing brain metastases and primary tumors of the breast from patients with breast cancer. UBR2 was expressed at significantly higher levels in brain metastases as compared to primary tumors of the breast.

\section{Discussion}

We recently reported that the deubiquitinases CYLD and USP2 were among the genes most differentially expressed in the brain metastases of patients with brain metastatic breast cancer. We report here that UBR2 was among the genes most differentially expressed in the brain metastases of patients with brain metastatic breast cancer. Together these data point to dysregulation of the ubiquitin system in central nervous metastases in patients with breast cancer.

UBR25-8 functions as an E3 ligase for substrates in the N-end rule pathway, wherein substrates with relatively short half-lives are targeted for proteasomal degradation through covalently modified by N-recognins: UBR E3 ligases like UBR2, via a degradation signal termed an N-degron at the aminoterminus of the substrate. As UBR2 was over-expressed in brain metastasis tissues relative to primary breast tumors, UBR2 small molecule inhibition can be explored as a means to pursuing novel therapeutic targets in patients with brain metastatic breast cancer. The substrates of UBR2 may be of relevance to the biology by which metastatic breast cancer cells home to and colonize the brain. 


\section{References}

1. Berndsen, C.E. and Wolberger, C., 2014. New insights into ubiquitin E3 ligase mechanism. Nature structural \& molecular biology, 21(4), p.301.

2. McMullin, R.P., Wittner, B.S., Yang, C., Denton-Schneider, B.R., Hicks, D., Singavarapu, R., Moulis, S., Lee, J., Akbari, M.R., Narod, S.A. and Aldape, K.D., 2014. A BRCA1 deficient-like signature is enriched in breast cancer brain metastases and predicts DNA damage-induced poly (ADP-ribose) polymerase inhibitor sensitivity. Breast Cancer Research, 16(2), pp.1-10.

3. Schulten, H.J., Bangash, M., Karim, S., Dallol, A., Hussein, D., Merdad, A., Al-Thoubaity, F.K., AlMaghrabi, J., Jamal, A., Al-Ghamdi, F. and Choudhry, H., 2017. Comprehensive molecular biomarker identification in breast cancer brain metastases. Journal of translational medicine, 15(1), p.269.

4. Bendell, J.C., Domchek, S.M., Burstein, H.J., Harris, L., Younger, J., Kuter, I., Bunnell, C., Rue, M., Gelman, R. and Winer, E., 2003. Central nervous system metastases in women who receive trastuzumab-based therapy for metastatic breast carcinoma. Cancer, 97(12), pp.2972-2977.

5. Ouyang, Y., Kwon, Y.T., An, J.Y., Eller, D., Tsai, S.C., Diaz-Perez, S., Troke, J.J., Teitell, M.A. and Marahrens, Y., 2006. Loss of Ubr2, an E3 ubiquitin ligase, leads to chromosome fragility and impaired homologous recombinational repair. Mutation Research/Fundamental and Molecular Mechanisms of Mutagenesis, 596(1-2), pp.64-75.

6. An, J.Y., Seo, J.W., Tasaki, T., Lee, M.J., Varshavsky, A. and Kwon, Y.T., 2006. Impaired neurogenesis and cardiovascular development in mice lacking the E3 ubiquitin ligases UBR1 and UBR2 of the Nend rule pathway. Proceedings of the National Academy of Sciences, 103(16), pp.6212-6217.

7. Xu, H., Shi, J., Yang, Z., Shao, F. and Dong, N., 2018. The N-end rule E3 ligase UBR2 activates Nlrp1b inflammasomes. bioRxiv, p.429225.

8. Wang, L., Mao, X., Ju, D. and Xie, Y., 2004. Rpn4 is a physiological substrate of the Ubr2 ubiquitin ligase. Journal of Biological Chemistry, 279(53), pp.55218-55223. 

breast cancer.

Rank of differential expression, probe ID, $p$-value with respect to differential expression, $t$, a moderated $t$ statistic, B, the log-odds of differential expression between the two groups compared, fold change (FC) of UBR2 expression between the brain metastases of patients with breast cancer and primary tumors of the breast, and gene and gene name are listed in this chart.

PAGE 5 
PAGE 6 


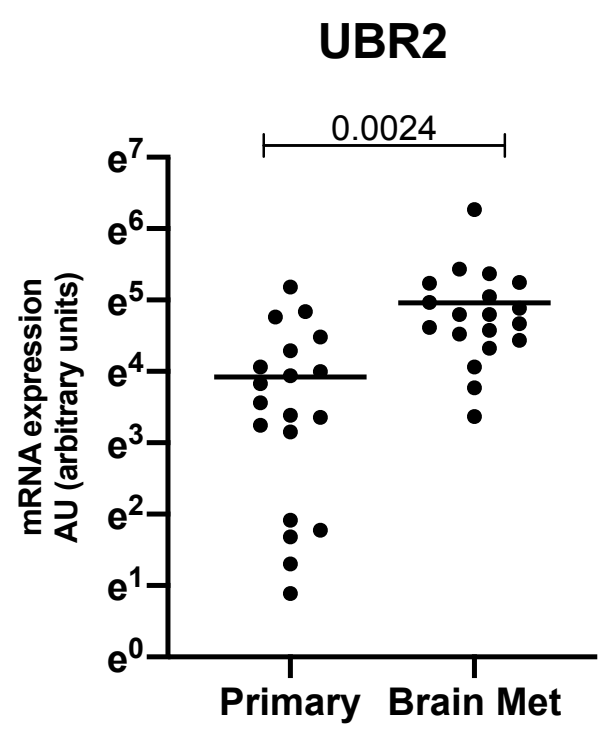

Figure 1: UBR2 is expressed at significantly higher levels in the brain metastases of patients with breast cancer as compared to primary tumors of the breast.

The mRNA expression level of UBR2 in primary tumors of the breast (left) and in the brain metastases of brain metastatic breast cancer patients (right) is graphically represented here with mean UBR2 levels marked and the result of a statistical test evaluating difference in mRNA expression level between brain metastases and primary tumors of the breast, a $p$-value, listed above. 